\title{
Erratum to: Analysis on the nonlinear response of cracked rotor in hover flight
}

\author{
Yongfeng Yang • Xingmin Ren • Weiyang Qin • \\ Yafeng Wu $\cdot$ Xizhe Zhi
}

Published online: 5 August 2010

(C) Springer Science+Business Media B.V. 2010

\section{Erratum to: Nonlinear Dyn (2010) 61: 183-192 DOI 10.1007/s11071-009-9640-7}

In the original publication, equations (5), (9) and (10) appearing on pages 185 and 186 should read as follows.

The equations of motion of the system can be written as

$$
\begin{aligned}
m \ddot{x}+ & 2 m c_{d} \omega_{t} \dot{x}+k_{z} x \\
& -f\left[\left(k_{z}-k_{11} \cos ^{2} \omega t-k_{22} \sin ^{2} \omega t\right) x\right. \\
& +\sin \omega t \cos \omega t\left(k_{22}-k_{11}\right) y \\
& +\left(-k_{13} \cos ^{2} \omega t-k_{24} \sin ^{2} \omega t\right) \theta_{y} \\
& \left.+\sin \omega t \cos \omega t\left(k_{24}-k_{13}\right) \theta_{x}\right] \\
= & m e \omega^{2} \sin \left(\omega t+\phi_{e}+\beta+\vartheta\right) \\
& +m r_{1} \omega_{1}^{2} \cos \vartheta-m g \sin \vartheta,
\end{aligned}
$$

The online version of the original article can be found under doi:10.1007/s11071-009-9640-7.

Y. Yang $(\varangle) \cdot$ X. Ren · W. Qin · Y. Wu · X. Zhi

Northwestern Polytechnical University, P.O. Box 264\#,

Xi' an 710072, People's Republic of China

e-mail: yyfnpu@hotmail.com

Y. Yang

e-mail: yyf@nwpu.edu.cn

$$
\begin{aligned}
m \ddot{y}+ & 2 m c_{d} \omega_{t} \dot{y}+k_{z} y-f\left[\sin \omega t \cos \omega t\left(k_{22}-k_{11}\right) x\right. \\
& +\left(k_{z}-k_{11} \sin ^{2} \omega t-k_{22} \cos ^{2} \omega t\right) y \\
& +\sin \omega t \cos \omega t\left(k_{24}-k_{13}\right) \theta_{y} \\
& \left.+\left(-k_{13} \sin ^{2} \omega t-k_{24} \cos ^{2} \omega t\right) \theta_{x}\right] \\
= & m e \omega^{2} \cos (\omega t+\phi+\beta+\vartheta)-m r_{1} \omega_{1}^{2} \sin \vartheta \\
& -m g \cos \vartheta \\
J_{d} \ddot{\theta}_{y} & +2 J_{d} c_{1} \omega_{s} \dot{\theta}_{y}-J_{p} \omega \dot{\theta}_{x}+k_{\theta} \theta_{y} \\
& -f\left[\left(-k_{13} \cos { }^{2} \omega t-k_{24} \sin ^{2} \omega t\right) x\right. \\
& +\sin \omega t \cos \omega t\left(k_{24}-k_{13}\right) y \\
& +\left(k_{\theta}-k_{33} \cos { }^{2} \omega t-k_{44} \sin ^{2} \omega t\right) \theta_{y} \\
& \left.+\sin \omega t \cos \omega t\left(k_{44}-k_{33}\right) \theta_{x}\right] \\
= & J_{p} \omega \omega_{1} \sin \vartheta \\
J_{d} \ddot{\theta}_{x} & +2 J_{d} c_{1} \omega_{s} \dot{\theta}_{x}+J_{p} \omega \dot{\theta}_{y}+k_{\theta} \theta_{x} \\
& -f\left[\sin \omega t \cos \omega t\left(k_{24}-k_{13}\right) x\right. \\
& +\left(-k_{13} \sin { }^{2} \omega t-k_{24} \cos ^{2} \omega t\right) y \\
& +\sin \omega t \cos \omega t\left(k_{44}-k_{33}\right) \theta_{x} \\
& \left.+\left(k_{\theta}-k_{33} \sin ^{2} \omega t-k_{44} \cos ^{2} \omega t\right) \theta_{y}\right] \\
= & J_{p} \omega \omega_{1} \cos \vartheta \\
&
\end{aligned}
$$

where $m$ is the mass of disc, $k_{z}$ is the bending stiffness of the uncracked rotor, and $k_{\theta}$ is the angular stiffness. $\phi_{e}$ is the original phase angle. $c_{d}$ and $c_{1}$ are transverse 
and swing vibration damps, respectively. $f$ is the crack model function. $\omega_{t}$ and $\omega_{s}$ are transverse and swing vibration critical speeds, respectively. $k_{i j}$ is the cross stiffness when the crack is open (the force in direction $i$ caused the unit bending in direction $j, 1,2,3$ and 4 represent the direction of $x, y, \theta_{y}$ and $\theta_{x}$, respectively) [1].

$$
\begin{aligned}
& X^{\prime \prime}+\frac{2 c_{d}}{\Omega} X^{\prime}+\frac{1}{\Omega^{2}} X-\frac{f}{\Omega^{2}} \\
& \times\left[\left(1-\breve{k}_{11} \cos ^{2} \tau-\breve{k}_{22} \sin ^{2} \tau\right) X\right. \\
& +\sin \tau \cos \tau\left(\breve{k}_{22}-\breve{k}_{11}\right) Y \\
& +\left(-\breve{k}_{13} \cos ^{2} \tau-\breve{k}_{24} \sin ^{2} \tau\right) \frac{\theta_{y}}{\delta} \\
& \left.+\sin \tau \cos \tau\left(\breve{k}_{24}-\breve{k}_{13}\right) \frac{\theta_{x}}{\delta}\right] \\
& =U \sin \left(\tau+\phi_{e}+\beta+\vartheta\right)+R_{1} \frac{\Omega_{1}^{2}}{\Omega^{2}} \cos \vartheta \\
& -\frac{1}{\Omega^{2}} \sin \vartheta, \\
& Y^{\prime \prime}+\frac{2 c_{d}}{\Omega} Y^{\prime}+\frac{1}{\Omega^{2}} Y-\frac{f}{\Omega^{2}}\left[\sin \tau \cos \tau\left(\breve{k}_{22}-\breve{k}_{11}\right) X\right. \\
& +\left(1-\breve{k}_{11} \sin ^{2} \tau-\breve{k}_{22} \cos ^{2} \tau\right) Y \\
& +\sin \tau \cos \tau\left(\breve{k}_{24}-\hat{k}_{13}\right) \frac{\theta_{y}}{\delta} \\
& \left.+\left(-\breve{k}_{13} \sin ^{2} \tau-\breve{k}_{24} \cos ^{2} \tau\right) \frac{\theta_{x}}{\delta}\right] \\
& =U \sin \left(\tau+\phi_{e}+\beta+\vartheta\right)-R_{1} \frac{\Omega_{1}^{2}}{\Omega^{2}} \sin \vartheta \\
& -\frac{1}{\Omega^{2}} \cos \vartheta \\
& \theta_{y}^{\prime \prime}+\frac{2 c_{1} \Omega_{s}}{\Omega} \theta_{y}^{\prime}-2 \theta_{x}^{\prime} \\
& -\frac{\Omega_{s}^{2} f}{\Omega^{2}}\left[\left(-\breve{k}_{13} \cos ^{2} \tau-\breve{k}_{24} \sin ^{2} \tau\right) X \delta\right.
\end{aligned}
$$

$+\sin \tau \cos \tau\left(\breve{k}_{24}-\breve{k}_{13}\right) Y \delta$

$+\left(1-\breve{k}_{33} \cos ^{2} \tau-\breve{k}_{44} \sin ^{2} \tau\right) \theta_{y}$

$\left.+\sin \tau \cos \tau\left(\breve{k}_{44}-\breve{k}_{33}\right) \theta_{x}\right]+\frac{\Omega_{s}^{2}}{\Omega^{2}} \theta_{y}$

$=2 \frac{\Omega_{1}}{\Omega} \sin \vartheta$,

$\theta_{x}^{\prime \prime}+\frac{2 c_{1} \Omega_{s}}{\Omega} \theta_{x}^{\prime}+2 \theta_{y}^{\prime}$

$-\frac{\Omega_{s}^{2} f}{\Omega^{2}}\left[\sin \tau \cos \tau\left(\breve{k}_{24}-\breve{k}_{13}\right) X \delta\right.$

$+\left(-\breve{k}_{13} \sin ^{2} \tau-\breve{k}_{24} \cos ^{2} \tau\right) Y \delta$

$+\sin \tau \cos \tau\left(\breve{k}_{44}-\breve{k}_{33}\right) \theta_{y}$

$\left.+\left(1-\breve{k}_{33} \sin ^{2} \tau-\breve{k}_{44} \cos ^{2} \tau\right) \theta_{x}\right]+\frac{\Omega_{s}^{2}}{\Omega^{2}} \theta_{x}$

$=2 \frac{\Omega_{1}}{\Omega} \cos \vartheta$

where

$\left(^{\prime}\right)=\frac{\mathrm{d}}{\mathrm{d} \tau}, \quad X=\frac{x}{\delta}, \quad Y=\frac{y}{\delta}, \quad \Omega=\frac{\omega}{\omega_{t}}$,

$\Omega_{s}=\frac{\omega_{s}}{\omega_{t}}, \quad \breve{k}_{i j}=\frac{k_{i j}}{k_{z}}, \quad \tau=\omega t$,

$U=\frac{e}{\delta}, \quad R_{1}=\frac{r_{1}}{\delta}, \quad \Omega_{1}=\frac{\omega_{1}}{\omega_{t}}$.

The equations were wrong by our carelessness when we were writing another paper and the simulation results of the original publication are acceptable.

\section{References}

1. Yang, Y.F., Ren, X.M., Qin, W.Y.: Answering two questions concerning stiffness of a Jeffcott rotor with transverse crack. J. Northwest. Polytech. Univ. 24, 778-781 (2006) 